\title{
Interaction law of 2D localized precession states
}

\author{
M. G. Clerc ${ }^{1}$, S. Coulibaly ${ }^{2(a)}$ and D. Laroze ${ }^{3(b)}$ \\ ${ }^{1}$ Departamento de Fúsica, FCFM, Universidad de Chile - Casilla 487-3, Santiago, Chile \\ ${ }^{2}$ Laboratoire de Physique des Lasers, Atomes et Molécules, CNRS UMR 8523, Centre d'Etudes et de Recherches Lasers \\ et Applications, Université des Sciences et Technologies de Lille - 59655 Villeneuve d'Ascq Cedex, France, EU \\ ${ }^{3}$ Instituto de Alta Investigación, Universidad de Tarapacá - Casilla 7D, Arica, Chile
}

received 9 February 2010; accepted in final form 3 May 2010

published online 1 June 2010

PACS 89.75.Kd - Patterns

PACS $75.10 . \mathrm{Hk}$ - Classical spin models

PACS 75.25.-j-Spin arrangements in magnetically ordered materials (including neutron and spin-polarized electron studies, synchrotron-source X-ray scattering, etc.)

\begin{abstract}
A theoretical study of the interaction of localized precession states on an easy-plane ferromagnetic layer submitted to a magnetic field that combines a constant and an oscillating part is reported. Within the framework the Landau-Lifshitz-Gilbert equation, we perform a comparison of analytical studies and micromagnetic simulations. Close to the parametric resonance, the parametrically driven damped nonlinear Schrödinger equation models this system. By means of this amplitude equation we are able to characterize the localized precession states and their pair interaction law. Numerically, we have a good agreement with the pair interaction law.
\end{abstract}

Copyright (C) EPLA, 2010

Introduction. - During the last years, emerging macroscopic particle-type solutions or localized states in macroscopic extended dissipative systems have been observed in different fields, such as: domains in magnetic materials, chiral bubbles in liquid crystals, current filaments in gas discharge, spots in chemical reactions, localized states in fluid surface waves, oscillons in granular media, isolated states in thermal convection, solitary waves in nonlinear optics, among others. Hence, one can infer the universality of the localized-states dynamics. Although these states are spatially extended, they exhibit properties typically associated with particles. Consequently, one can characterized them with a family of continuous parameters such as position, amplitude and width. This is exactly the type of description used in more fundamental physical theories like quantum mechanics and particle physics. However, localized states emerging in extended dissipative systems are characterized by being made of a large number of atoms or molecules (of the order of Avogadro's number) that behave coherently. The paradigmatic example of macroscopic localized states are solitons reported in the context of fluid dynamics, nonlinear optics and Hamiltonian systems [1]. The

\footnotetext{
(a) E-mail: saliya. coulibaly@phlam.univ-lille1.fr

(b) Current address: Max Planck Institute for Polymer Research 55021 Mainz, Germany, EU.
}

solitons arise from a robust balance between dispersion and nonlinearity. The generalization of this concept to dissipative and out-of-equilibrium systems has led to several studies in the last decades, in particular to the definition of localized structures intended as patterns appearing in a restricted region of space $[2,3]$.

In one-dimensional systems, localized states can be described, geometrically speaking, as spatial trajectories that connect a steady state with itself, that means, they are homoclinic orbits from the viewpoint of the dynamicalsystems theory (see the review [4] and references therein), while domain walls or fronts are seen as spatial trajectories joining two different steady states - heteroclinic curvesof the corresponding spatial dynamical system [5]. In a particular case the localized patterns can be understood as homoclinic orbits in the Poincaré section of the corresponding spatial-reversible dynamical system [4-7]. The particular case of localized patterns can also be understood as a consequence of the interaction of fronts with oscillatory tails $[8,9]$; the characterization of such a localized structure is well known and details can be found in $[10,11]$. All the aforementioned scenario cannot be extended to localized states in two-dimensional space systems, which currently lack an equivalent geometrical description as those developed in the one-dimensional systems [4]. There is another type of stabilization mechanism that generates localized structures without 


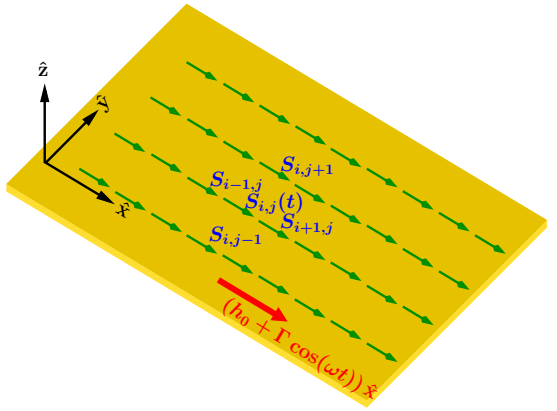

Fig. 1: (Colour on-line) Schematic representation of an uniaxial anisotropic Heisenberg ferromagnetic layer. Small arrows represent the local magnetization $\left(\mathbf{S}_{i, j}\right)$ and the large arrow stands for the external magnetic field.

oscillatory tails based on non-variational effects [12], where the fronts interaction is led by the non-variational terms [13]. Localized structures that we shall study are of non-variational type.

A characteristic property of particle-type solutions is that their interaction can be described simply in terms of continuous parameters describing the localized states. Recently, improved experimental techniques have increased the interest in the study of interaction of localized states $[14,15]$. The understanding of the pair interaction law of localized states allows us to comprehend the evolution of the system to equilibrium; more importantly, we expect a more efficient management and control of these localized states for their potential applications. This has a particular technological interest in new storage media like semiconductor cavity [16]. One of the most prominent parametric oscillators in the context of spintronics are the nanopillars [17], the ability to generate and study the localized structures and the interaction between them opens up the possibility of developing new magnetic devices.

The aim of this letter is to study, in the classical spin limit, the interaction of localized precession states on an easy-plane ferromagnetic spin layer submitted to an external magnetic field that combines a constant and an oscillating part. This magnetic system is described phenomenologically by the Landau-Lifshitz-Gilbert equation. In this framework we perform a comparative study of analytical results and micromagnetic simulations. Close to the parametric resonance, the parametrically driven damped nonlinear Schrödinger equation models this system. By means of this amplitude equation we are able to characterize the pair interaction law between localized excitations. Numerically, we have a good agreement with the proposed pair interaction law.

Parametrically driven magnetic layer. - Let us consider a two-dimensional anisotropic Heisenberg ferromagnetic layer formed by $N_{x} \times N_{y}$ spins or magnetic moment exposed to an external magnetic field, which is contained in the plane $(x, y)$ and oriented in the direction $\hat{\mathbf{x}} \equiv(1,0,0)$. Figure 1 depicts the setup of the system under study. When the quantum effects are small enough, the spin vectors $\mathbf{S}_{i, j}$ can be treated as classical spin or magnetic moment [18] and satisfies the dynamical evolution $\dot{\mathbf{S}}_{i, j}=-\gamma \mathbf{S}_{i, j} \times\left(\partial \mathcal{H} / \partial \mathbf{S}_{i, j}\right)[19]$, where $\gamma$ is the gyromagnetic constant and the Hamiltonian $\mathcal{H}$ has the form $[20]$

$$
\begin{aligned}
\mathcal{H}= & \sum_{i, j}^{N}\left(-J_{x} \mathbf{S}_{i, j} \mathbf{S}_{i+1, j}-J_{y} \mathbf{S}_{i, j} \mathbf{S}_{i, j+1}\right. \\
& \left.+2 D\left(S_{i, j}^{z}\right)^{2}-g \mu\left(S_{i, j}^{x}\right) H_{x}\right) .
\end{aligned}
$$

Here, $\left\{J_{x}, J_{y}\right\}$ are the exchange coupling constants which are of the same order of magnitude, $H_{x}$ and $D$ account for the external magnetic field and the anisotropy energy, respectively. The characterization and the understanding of this discrete system is a complicated task. Hence we study the continuum limit of this set of the ordinary differential equations [18,21]. Thus we can assume that $\mathbf{S}_{i, j}(t) \rightarrow \mathbf{S}(\vec{r}, t)$, where $\vec{r}(x, y)$ accounts for the coordinates describing the magnetic plane and taking the limit $J_{x} \gamma\left(\mathbf{S}_{i+1, j}-2 \mathbf{S}_{i, j}+\mathbf{S}_{i-1, j}\right)+$ $J_{y} \gamma\left(\mathbf{S}_{i, j+1}-2 \mathbf{S}_{i, j}+\mathbf{S}_{i, j-1}\right) \rightarrow\left(l_{x} \partial_{x}^{2}+l_{y} \partial_{y}^{2}\right) \mathbf{S}$, scaling the spatial coordinates $\left(l_{x} \partial_{x}^{2}+l_{y} \partial_{y}^{2}\right) \mathbf{S} \rightarrow l_{e x} \nabla_{\perp}^{2} \mathbf{S}$, where $l_{e x}$ denotes the characteristic interaction length and $\nabla_{\perp}^{2}$ is the Laplacian operator in the new transversal coordinates $\left(\nabla_{\perp}^{2} \equiv \partial_{x x}+\partial_{y y}\right)$. Finally, introducing phenomenologically the Gilbert damping, the motion of the magnetization field is governed by the well-known LandauLifshtiz-Gilbert equation

$$
\partial_{\tau} \mathbf{M}=\mathbf{M} \times\left[\nabla_{\perp}^{2} \mathbf{M}-\beta(\mathbf{M} \cdot \hat{z}) \hat{z}+\mathbf{H}_{e}-\alpha \partial_{\tau} \mathbf{M}\right],
$$

where $\mathbf{M} \equiv \mathbf{S} / M_{s}$ stands for the unit vector of the magnetization, with $M_{s}$ the saturation magnetization; we have also considered the following normalization of scales and parameters $\left\{\tau \rightarrow \gamma M_{s} t, \beta \rightarrow 4 D / \gamma M_{s}, \mathbf{H}_{e} \rightarrow g \mu \mathbf{H} / \gamma M_{s}\right\}$, where $\beta>0$ is the uniaxial easy-plane anisotropy constant, and $\alpha$ is the damping parameter. This damping parameter for a great quantities of materials is small. Assuming that the external magnetic field is constant and neglecting damping effects $(\alpha=0)$, the dynamics of model (2) is time reversible. However, when we consider the effects of damping and an external magnetic field with both a constant and an oscillatory part, model (2) becomes a quasireversible type, i.e. a time reversible system perturbed with injection and dissipation of energy. The instabilities of these types of systems have been studied during the last decades [22].

As a result of the anisotropy and constant external field $\left(\mathbf{H}_{e}=h_{0} \hat{\mathbf{x}}\right)$, the natural equilibrium of the previous model (2) corresponds to the magnetization field lying in the direction of the external magnetic field, $\mathbf{M}=\hat{\mathbf{x}}$ (cf. fig. 1). When spatial coupling is ignored, it is easy to show that the dynamics around this equilibrium is described by a nonlinear oscillator with natural frequency $\omega_{0}=\sqrt{h_{0}\left(\beta+h_{0}\right)}[23,24]$. It is worthy to note that in 


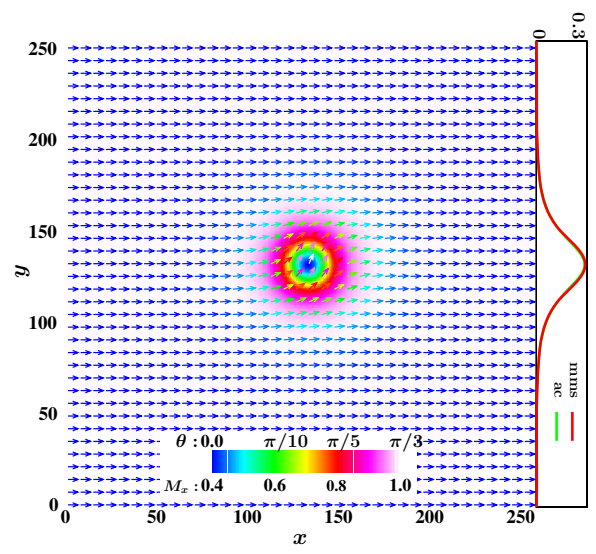

Fig. 2: (Colour on-line) Localized precession states obtained from micromagnetic simulation (mms) of model (2) by $\beta=1$, $h_{0}=0.1, \alpha=0.01, \Gamma=0.009$ and $\omega=0.328$. The background color describes the value of the intensity of $M_{x}$. The arrows represent the instantaneous local magnetization unit vector $\mathbf{M}$. Its respective color scale accounts for the value of the angle $\theta \equiv \widehat{(\hat{\mathbf{x}}, \mathbf{M})}$. The inset shows a profile of $M_{y}$ and is compared with the variational approximation (ac) given by (5).

eq. (2), the magnetization components are proportional to the external magnetic field, which therefore acts as a parametric forcing. Then if this field combines a constant and a time-periodic part $\left(\mathbf{H}_{e}=\left[h_{0}+\Gamma \cos (\omega t)\right] \hat{\mathbf{x}}\right)$ oscillating about twice the natural frequency $\left(\omega \equiv 2\left(\omega_{0}+\nu\right)\right.$, where $\nu$ is the detuning parameter $)$, the system exhibits a parametric resonance at $\Gamma^{2}\left(\beta / 4 \omega_{0}\right)^{2}=\alpha^{2}\left(\beta / 2+h_{0}\right)^{2}+\nu^{2}$ for small $\left\{\nu, h_{0}, \alpha, \Gamma\right\}$-Arnold's tongue. Dynamically speaking this resonance corresponds to an undamped precession of the magnetization unit vector around the direction of the external magnetic field with the angular velocity $\omega_{0}$.

Localized precession states. - The inclusion of spatial coupling should increase the complexity of the dynamics. For example, one expects the formation of patterns, domain walls, and localized states near the parametric resonance. This wide range of phenomena is described in a unified manner near the parametric resonance by the parametrically driven damped nonlinear Schrödinger equation [25]. In order to show that the LLG system can exhibit this kind of solutions, we have numerically solved (2) using the fixed step fourth-order RungeKutta scheme $\left(\mathrm{d} t=10^{-4}\right)$, over a square lattice of size $250 \times 250$ a.u. with spatial a discretization step set to 0.1 in both directions. Numerical solutions of (2) are simultaneously compared with those obtained by integration of the equivalent stereographic representation [26] in order to check their accuracy. Figure 2 illustrates the typical non-propagative localized precession states obtained from these simulations. This magnetic state, is characterized by a localized precession surrounded by a static magnetic state that lies in the $x$-direction.

Changing the detuning and the amplitude of the forcing, the system exhibits a stable uniform precession state,

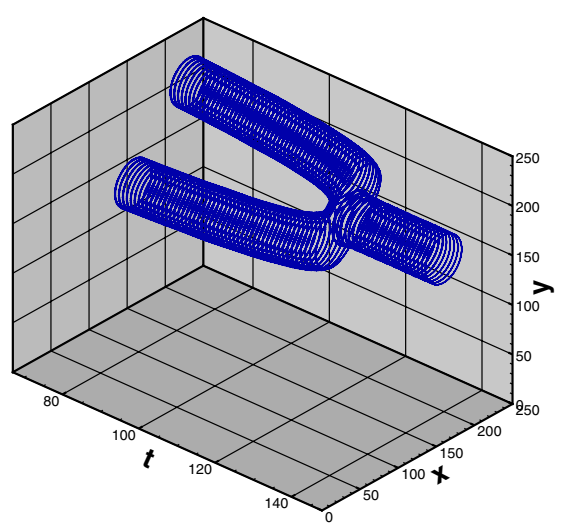

Fig. 3: (Colour on-line) Space-temporal diagram of interaction of a pair of localized precession states obtained from model (2) by the same parameters of fig. 1 . The circles represent the core of the localized states, i.e. the isolines of the full width at half maximum of the localized state.

which exhibits a spatial instability when the detuning is decreased. This instability is characterized initially by the appearance of a pattern with a well-defined wavelength, which then decomposes into a gas of localized precession states. The subsequent dynamics of this system is led by the interaction of these states. This interaction is characterized by the collision of a pair of localized states, ended by a coalesce process from which there emerges a single localized precession state. In fig. 3 this process is shown.

Parametrically driven damped nonlinear Schrödinger equation. - To understand the localized precession state and the interaction between them, in the quasi-reversible limit $\left(\Gamma \sim \nu \sim \alpha \ll \omega_{0}\right)$ and close to the parametric resonance, we can introduce the following Ansatz $[23,24]$ into eq. (2):

$$
\begin{aligned}
& M_{x} \approx 1-\frac{M_{y}^{2}+M_{z}^{2}}{2}, \\
& M_{y} \approx \frac{1}{h_{0}}\left[1+\frac{\Gamma}{h_{0}}\right] \dot{M}_{z}, \\
& M_{z} \approx 4 \sqrt{\frac{\omega_{0} h_{0}}{\beta\left(\omega_{0}^{2}+3 h_{0}^{2}\right)}} \psi(\vec{r}, t) e^{i\left(\omega_{0}+\nu\right) t}+c . c .,
\end{aligned}
$$

to the dominant order. After straightforward calculations and imposing a solvability condition for the corrections of the previous Ansatz we find (the parametrically driven damped nonlinear Schrödinger equation)

$$
\partial_{t} \psi=-i \nu \psi-i|\psi|^{2} \psi-i \nabla_{\perp}^{2} \psi-\mu \psi+\gamma \bar{\psi}
$$

with $\gamma \equiv \beta \Gamma / 4 \omega_{0}$ and $\mu \equiv\left(\beta / 2+h_{0}\right) \alpha$. This model has been derived in several contexts to describe pattern and localized structures, such as vertically oscillating layers of water [27], nonlinear lattices [28], optical fibers [29], Kerrtype optical parametric oscillators [30], among others. 
It is well known that eq. (4) exhibits stable nonpropagative dissipative solitons in two spatial dimensions [31]. The localized states have the form $\psi= \pm R_{s}(r=|\vec{r}|) e^{i \theta_{0}}$, where $\cos \left(2 \theta_{0}\right)=\mu / \gamma$, and $R_{s}$ satisfies the equation $\partial_{r r} R_{s}+\partial_{r} R_{s} / r-\lambda R_{s}+R_{s}^{3}=0$, where $\lambda \equiv-\nu+\sqrt{\gamma^{2}-\mu^{2}}>0$. To our knowledge, there does not exist an analytical solution of the localized state. However the asymptotic behaviors of this solution are well defined: for instance $R(r \rightarrow \infty) \rightarrow e^{-\sqrt{\lambda} r} / \sqrt{r}$. Furthermore, using the variational method, one can also obtain a good approximation by [32]

$$
R_{s}(r)=A_{0} \sqrt{\lambda} \operatorname{sech}\left(B_{0} \sqrt{\frac{\lambda}{2}} r\right),
$$

where $A_{0}=2.166$ and $B_{0}=1.32$. From this variational approach and using the Ansatz (3), one can have an adequate representation for the localized precession states. In inset of fig. 2, we contrasted this approach with those obtained from micromagnetic simulations of the model (2). We note that there is a quite good agreement.

From the approximated localized state (5), one can infer that for negative detuning, this solution appears by a saddle-node bifurcation when dissipation and energy injection are equal $(\gamma=\mu$ and $\nu<0)$. Furthermore, this solution is unstable when the uniform magnetization $\mathbf{M}=\hat{\mathbf{x}}$ - which supports this localized state - becomes unstable at Arnold's tongue $\left(\gamma^{2}=\nu^{2}+\mu^{2}\right.$, by $\left.\nu<0\right)$. The characteristic size and amplitude of the localized precession states, respectively, are $1 / \sqrt{\lambda}$ and $\sqrt{\lambda}$.

Pair interaction law of localized states. - We consider two localized states sufficiently separated, i.e. the distance between them is greater than the characteristic size of the localized states. Hence, we can introduce the following Ansatz: $\psi(r, t)=R(r, t) e^{i \theta(r, t)}$,

$$
\begin{aligned}
R & =R_{s}^{+}\left(r+\frac{\Delta(t)}{2} \hat{r}\right)+\chi R_{s}^{-}\left(r-\frac{\Delta}{2} \hat{r}\right)+\rho(\vec{r}, \Delta), \\
\theta & =\theta_{0}+\varphi(\vec{r}, \Delta),
\end{aligned}
$$

where $R_{s}^{ \pm}$are non-propagative dissipative solitons, $\Delta(t)$ stands for the distance between the localized states $(\Delta \gg$ $\sqrt{\lambda}), \hat{r}$ is the unit vector in the direction between the localized states, $\chi= \pm 1$ is a sign that defines whether the solutions are in or out of phase, $\rho(\vec{r}, \Delta)$ and $\varphi(\vec{r}, \Delta)$ are, respectively, the corrections functions. Defining $W \equiv$ $R_{s}^{+}+\chi R_{s}^{-}$, considering the parameter region where the dissipation and injection of energy are similar $(0<\gamma-$ $\mu \ll 1$ ), introducing the above Ansatz into eq. (4) and linearizing correction functions, we obtain

$$
\begin{gathered}
W \partial_{t} W=\vec{\nabla}_{\perp}\left(W^{2} \vec{\nabla}_{\perp} \varphi\right), \\
W \partial_{t} \varphi=\mathcal{L} \rho-2 \mu \varphi-3 \chi R_{s}^{+} R_{s}^{-} W,
\end{gathered}
$$

where $\mathcal{L} \equiv-\nu+\sqrt{\gamma^{2}-\mu^{2}}+3 W^{2}+\vec{\nabla}_{\perp}^{2}$ is a linear operator.

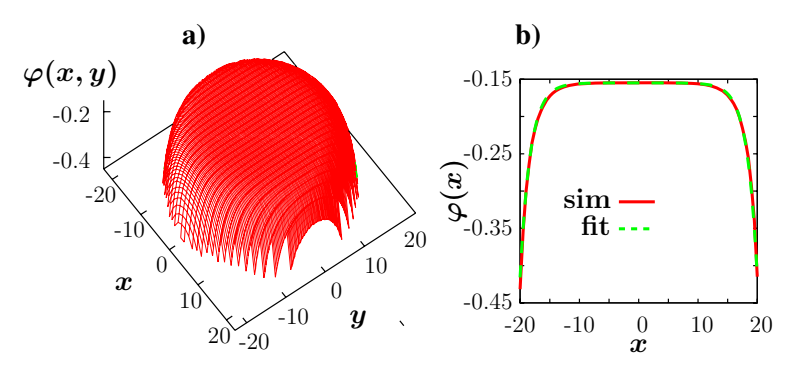

Fig. 4: (Colour on-line) Phase $\varphi$ around one of the two interacting localized solitons of eq. (4) using $\gamma=0.53, \mu=0.25$ and $\nu=0.05$. a) two-dimensional representation, b) solid and dashed lines are, respectively, the profile of $\varphi$ obtained from the numerical simulation of model (4) and fit (9).

To solve the above equations, we need to derive $\varphi$. However finding a global solution for $\varphi$ remains a difficult task. Nevertheless, if localized states are diluted we can find $\varphi$ around the center of each dissipative soliton. In order to get $\varphi$, we changed our reference system by $\vec{r}^{\prime}=\vec{r}+\hat{r} \Delta / 2$, and in consequence, we approximate the functions $W\left(r^{\prime}=\left|\vec{r}^{\prime}\right|\right) \approx R_{s}^{+}\left(r^{\prime}\right)$ and $\partial_{t} W \approx \dot{\Delta} \partial_{r^{\prime}} R_{s}^{+} / 2$. Next, using the approximation (5) and integrating eq. (7), after straightforward calculations we find out that

$$
\varphi\left(r^{\prime}\right)=-\frac{\dot{\Delta}}{4}\left(r^{\prime}-\frac{1}{2 B} \operatorname{Shi}\left(2 B r^{\prime}\right)\right) \equiv \dot{\Delta} \Theta\left(r^{\prime}\right),
$$

where $\operatorname{Shi}\left(2 B r^{\prime}\right)$ is the hyperbolic sine integral. Figure 4 compares this approximation with those obtained from numerical simulations.

Replacing expression (9) into eq. (8), we find a linear equation in $\rho$. To solve this linear equation, we use the Fredholm alternative [2]. Therefore we introduce the following inner product: $\langle f \mid g\rangle=\iint f g \mathrm{~d} x \mathrm{~d} y$, where $\mathcal{L}$ is self-adjoint and its kernel is characterized by $\left\{\partial_{r^{\prime}} R_{s}^{ \pm}\right\}$. Then applying this product to eq. (8), we find the following solvability condition (pair interaction law):

$$
\begin{aligned}
\dot{\Delta} & =-\chi \frac{3}{a \mu} \int_{0}^{r^{\prime}} \partial_{r^{\prime}} R_{s}^{-}\left(R_{s}^{-}\right)^{2} R_{s}^{+}\left(\hat{\mathbf{r}}^{\prime}-\Delta \hat{\mathbf{x}}\right) r^{\prime} \mathrm{d} r^{\prime} \mathrm{d} \theta \\
& \approx-\chi \frac{b}{a \mu} \frac{e^{-\sqrt{\lambda} \Delta}}{\sqrt{\Delta}}
\end{aligned}
$$

where $\quad a=\left\langle\partial_{r^{\prime}} R_{s}^{-} \mid \Theta\right\rangle \quad$ and $\quad b \equiv 3 A \int_{0}^{r^{\prime}} \partial_{r^{\prime}} R_{s}^{-}\left(R_{s}^{-}\right)^{2}-$ $e^{-\sqrt{\lambda} r \cos \theta} \mathrm{d} r^{\prime}$. The pair interaction law is derived using the asymptotic behavior of the localized state. Hence, the localized precession states experience an exponential force of attraction $(\chi=1)$ or repulsion $(\chi=-1)$ if they are in or out of phase. In the limit of diluted localized states the prefactor $1 / \sqrt{\Delta}$ is a correction of the dominant term which is exponential. However, when these states are located closer to this prefactor it becomes more relevant.

Hence the interaction of localized precession states is like over-damped two-dimensional particles with two types of charges where the interaction is only radial. In fig. 5 the evolution of the distance between the localized states 

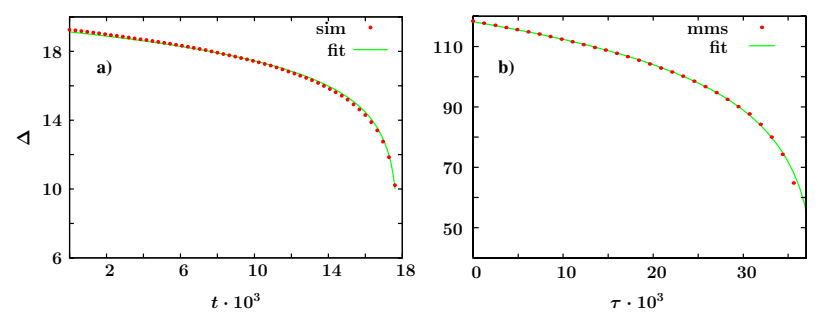

Fig. 5: (Colour on-line) Temporal evolution of the separation distance $\Delta(t)$. The points represent the separation distance obtained from a) micromagnetic simulations of the model (2) using the parameters of fig. 2 and b) numerical simulations of eq. (4), using the parameters of fig. 4. The solid line is the evolution of the separation distance deduced from the interaction law (10).

obtained using the pair interaction law is compared to those obtained from the numerical simulations of the parametrically driven damped nonlinear Schrödinger equation and micromagnetism simulations of the model (2). We find a remarkable agreement.

In the case of reversible two-dimensional systems, using Lagrangian methods one can derive a similar interaction law, however the radial dynamics can be enhanced by the appearance of tangential forces [33,34].

Conclusions. - We have theoretically studied the interaction of localized precession states on an easy-plane ferromagnetic spin layer submitted to a magnetic field that combines a constant and an oscillating parts. We have performed a comparison of analytical studies and micromagnetic simulations. Close to the parametric resonance, we are able to characterize the localized precession states and their pair interaction law. Numerically, we have a good agreement with the pair interaction law.

Given the numerical parameters that we have considered in the micromagnetic simulations in the case of permalloy $\left(M_{s} \simeq 10 \mathrm{kOe}\right)$ and $\mathrm{Ni}\left(M_{s} \simeq 6.2 \mathrm{kOe}\right)$ the characteristic size of the localized precession state is $43 \mathrm{~nm}$ and $57 \mathrm{~nm}$, respectively. Therefore the description presented here corresponds to a particular-type state of nanoscale.

The simulation software $\operatorname{Dim} X$ developed at INLN, France, has been used for all the numerical simulations. MGC acknowledges the financial support of FONDECYT grant No. 1090045. DL thanks the partial financial support from FONDECYT No. 11080229, Millennium Scientific Initiative, P06-022-F, Financiamiento Basal para Centros Científicos Y Technológicos de Excelencia CEDENNA and Convenio de Desempeño UTA/Mineduc.

\section{REFERENCES}

[1] Newell A. C., Solitons in Mathematics and Physics (Society for Industrial and Applied Mathematics, Philadelphia) 1985.
[2] Pismen L. M., Patterns and Interfaces in Dissipative Dynamics, in Springer Series in Synergetics (Springer, Berlin Heidelberg) 2006.

[3] Cross M. and Greenside H., Pattern Formation and Dynamics in Nonequilibrium Systems (Cambridge University Press, New York) 2009.

[4] Coullet P., Int. J. Bifurcat. Chaos, 12 (2002) 2445.

[5] van Saarloos W. and Hohenberg P. C., Phys. Rev. Lett., 64 (1990) 749.

[6] Woods P. D. and Champneys A. R., Physica D, 129 (1999) 147.

[7] Hunt G. W., Lord G. J. and Champneys A. R., Comput. Methods Appl. Mech. Eng., 170 (1999) 239.

[8] Clerc M. G. and Falcon C., Physica A, 356 (2005) 48.

[9] Bortolozzo U., Clerc U., Falcon C., Residori S. and Rojas R., Phys. Rev. Lett., 96 (2006) 214501.

[10] Kawasaki K. and Отна T., Physica A (Amsterdam), 116 (1982) 573.

[11] Caroli B., Caroli C. and Fauve S., J. Phys. I, 2 (1992) 281.

[12] Thual O. and Fauve S., J. Phys. (Paris), 49 (1988) 1829; Phys. Rev. Lett., 64 (1990) 282.

[13] Hakim V. and Pomeau Y., Eur. J. Mech. B/Fluids, Suppl., 10 (1991) 137.

[14] Buchanan K. S. et al., Nat. Phys., 1 (2005) 172.

[15] Rotschild C. et al., Nat. Phys., 2 (2006) 769.

[16] Barland S. et al., Nature, 419 (2002) 699.

[17] Yang Z., Zhang S. and Li Y. C., Phys. Rev. Lett., 99 (2007) 134101.

[18] Mikeska H. J., J. Phys. C, 11 (1978) L29.

[19] Kosevich A. M., Ivanov B. A. and Kovalev A. S., Phys. Rep., 194 (1990) 117.

[20] Kittel C., Rev. Mod. Phys., 21 (1949) 541.

[21] Herring C. and Kittel C., Phys. Rev., 81 (1951) 869.

[22] Clerc M., Coullet P. and Tirapegui E., Phys. Rev. Lett., 83 (1999) 3820; Int. J. Bifurcat. Chaos, 11 (2001) 591; Opt. Commun., 166 (1999) 159; Phys. Lett. A, 287 (2001) 198.

[23] Barashenkov I. V., Bogdan M. M. and Korobov V. I., Europhys. Lett., 15 (1991) 113.

[24] Clerc M. G., Coulibaly S. and Laroze D., Phys. Rev. E, 77 (2008) 056209; Int. J. Bifurcat. Chaos, 19 (2009) 3525; Physica D, 239 (2010) 72.

[25] Coullet P., Frisch T. and Sonnino G., Phys. Rev. E, 49 (1994) 2087.

[26] Lakshmanan M. and Nakamura K., Phys. Rev. Lett., 53 (1984) 2497 and references therein.

[27] Miles J. W., J. Fluid Mech., 148 (1984) 451; Zhang W. and Viñals J., Phys. Rev. Lett., 74 (1995) 690; Wang X. and Wei R., Phys. Rev. E, 57 (1998) 2405; ClerC M. G. et al., Philos. Trans. R. Soc. A, 367 (2009) 3213.

[28] Denardo B. et al., Phys. Rev. Lett., 68 (1992) 1730.

[29] Kutz J. N. et al., Opt. Lett., 18 (1993) 802.

[30] Longhi S., Phys. Rev. E, 53 (1996) 5520.

[31] Barashenkov I. V., Alexeeva N. V. and Zemlyanaya E. V., Phys. Rev. Lett., 89 (2002) 104101.

[32] Anderson D., Bonnedal M. and Lisak M., Phys. Fluids, 22 (1979) 1838.

[33] Steblina V. V., Kivshar Y. S. and Buryak A. V., Opt. Lett., 23 (1998) 156.

[34] Gorshkov K. A. and Ostrovsky L. A., Physica D, 3 (1981) 428. 